\title{
Evaluation of Hematological Scoring System (HSS) for Early Diagnosis of Neonatal Sepsis
}

\author{
M M Yusuf ${ }^{1}$, B H N Yasmeen ${ }^{2}$, M A K A Chowdhury ${ }^{3}$
}

\begin{abstract}
Background : Neonatal sepsis is a common occurrence and major health problem in Bangladesh and other developing countries, a devastating cause of morbidity and mortality during first 28 days of life.

Objective: This study was carried out to evaluate the efficacy of hematological scoring system (HSS) in the early detection of neonatal sepsis.

Study period : June 2014 to December 2014(Six months).

Methods : This was a prospective study consisting of 218 neonates admitted at Intensive Care Unit (ICU) Dhaka Shishu Hospital who were clinically suspected to have septicaemia. Newborn babies with major congenital anomaly,inborn error of metabolism, severely jaundiced due to blood group incompatibilities or RDS (due to surfactant deficiency) were excluded. The hematological parameter were measured in all cases. Patients were scored according to hematological scoring system (HSS), incorporating increase or decrease in leucocytes count, absolute neutrophil count, immature neutrophil count (e.g. band form), presence of degenerative changes in neutrophil (e.g. toxic granulation, vacuolation). CRP estimation and blood culture were also performed. Blood culture is considered as gold standard for diagnosis of sepsis. Fourteen out of $128(11 \%)$ had culture proven sepsis. They were predominantly preterm and of very low birth weight. Score of 3 was considered as positive.

Results : On evaluation of various hematological parameters the HSS was found to have a sensitively of $71 \%$, specificity of $73 \%$, positive predictive value $24 \%$, negative predictive value $95 \%$.

Conclusion: HSS is a simple, quick, readily available effective tool to detect early neonatal sepsis. Considering the high sensitivity, specificity, negative predictive value, this study implies that score 33 were reliable as a screening tool for early neonatal sepsis.
\end{abstract}

Key words : Neonatal sepsis, Blood culture, Hematological Scoring System

Northern International Medical College Journal Vol. 7 No. 02 January 2016, Page 135-138

1 Dr. Mir Mohammad Yusuf Registrar

Dhaka Shishu (Children) Hospital

${ }^{2}$ Prof. Dr. B H Nazma Yasmeen

Professor and Head

Dept. of Paediatrics

Northern International

Medical College

${ }^{3}$ Prof. Dr. M A K Azad Chowdhury

Professor and Head

Dept. of Neonatology

BICH, Dhaka Shishu Hospital

\section{Correspondence}

Dr. Mir Mohammad Yusuf

Registrar, Dhaka Shishu (Children)

Hospital, Dhaka

Email : dr.miryusufpavel@gmail.com

\section{Introduction}

Neonatal sepsis is defined as systemic inflammatory response of the body to an infection during first 28 days of life diagnosed either on microbiologic cultures or strong clinical evidence of an infection. Upto $25 \%$ of children in intensive care units have sepsis. ${ }^{1}$ Worldwide neonatal sepsis is responsible for 4 million deaths annually, most of them occur in developing countries ${ }^{2}$ where mortality rate is between 11-68 per 1000 live birth and in Bangladesh rate is 42 per 1000 live birth. ${ }^{3}$ In developed countries about 5 per 1000 live birth. ${ }^{4}$ Group B streptococcal (GBS) disease is the most important cause of neonatal sepsis in Europe, but there is preponderance of gram negative organism in tropical and developing countries. ${ }^{5}$ It has been seen that gram negative organism are leading pathogen in Bangladesh. ${ }^{6}$ Apart from mortality, morbidity associated with neonatal sepsis includes sensorineural hearing loss, visual disturbances, seizures, and neurodevelopmental issues. $^{7}$ This life-threatening condition is treatable if diagnosed early but unfortunately, the early signs and symptoms are often nonspecific and confusing which makes it difficult to establish an early clinical diagnosis. ${ }^{8}$ As a result of this uncertainty antibiotics are often started on the slightest clinical suspicions of sepsis. This approach is effective in fighting against the acute infections, but increases the risks of antibiotics side effects and the emergence of drug resistant organisms in neonatal units. 8,9 Blood culture is considered as gold standard for diagnosis of sepsis. ${ }^{10} \mathrm{~A}$ drawback of culture based diagnosis is the assay time of up to 36 hours. ${ }^{10}$ After 36 hours of incubation period, sensitivity and specificity are $100 \% .{ }^{11}$ Measures of acute phase proteins, 
cytokines, cell surface antigens, and bacterial genomes have been used, either alone or in combination, for early diagnosis of neonatal sepsis. Some of these markers are sensitive and specific, but sophisticated or expensive so impractical for developing countries like ours. ${ }^{8}$ Early diagnosis of neonatal sepsis is still a great challenge. Many attempts have been made to develop a set of screening tests for early and rapid diagnosis of the infected neonates.

Therefore the ideal diagnostic test should have quick results, inexpensive and adequate sensitivity, specificity, positive predictive value and negative predictive value to reliably exclude sepsis and avoid unnecessary antibiotic therapy. In this regards early diagnosis of neonatal sepsis a hematological scoring system (HSS) of Rodwell are preferable because it includes all parameters. Hematological scoring system (HSS) that we studied includes complete blood picture and $\mathrm{C}$ reactive protein estimation (CRP) which is quick and cost effective.

\section{Patients and Methods}

This prospective study was conducted at intensive care unit, department of pediatrics, Dhaka Shishu Hospital during the period of June 2014 to December 2014. All the newborns aged 0 to 28 days presented with maternal history of premature rupture of membranes, intrapartum fever ${ }^{3} 101^{0} \mathrm{~F}$ or clinical signs of sepsis namely; neonatal fever ${ }^{3} 101^{0} \mathrm{~F}$ or hypothermia, poor feeding and lethargy or depressed neonatal reflexes were included in this study. Neonates who presented with major congenital anomaly, inborn error of metabolism, severely jaundiced due to blood group incompatibilities or RDS due to surfactant deficiency were excluded from this study. After taking a careful history previously designed and developed questionnaire was filled up with detailed information and recorded by the investigator. With all aseptic precaution at least $2 \mathrm{ml}$ of blood was withdrawn from suspected sepsis patients within 24 hours of admission. $1 \mathrm{ml}$ sample was anticoagulated with EDTA and using automated hematology analyzer, values of total leucocyte count (TLC), differential leucocyte count and platelet count were noted. Peripheral blood smears were stained by Leishman method. $1 \mathrm{ml}$ of blood was inoculated aseptically into conventional blood culture bottle sent to department of microbiology, Dhaka Shishu Hospital for culture and sensitivity. Patient was scored according to hematological scoring system (HSS), incorporating increase or decrease in leukocyte count, absolute neutrophil count, immature PMNs count and platelet count. Degenerative changes in PMNs (toxic granulation and vacuolation) which have seen with Giemsa stained slides. CRP estimation was also performed (Table I). Score of 3 was considered as positive.
Table I : Hematological Scoring System

\begin{tabular}{|l|l|c|}
\hline \multicolumn{1}{|c|}{ Hematological test } & \multicolumn{1}{|c|}{ Abnormality } & Score \\
\hline Increased or decreased WBC count & $\begin{array}{l}\text { At birth } 5,000 \mathrm{~m} \mathrm{~m}^{3} \text { or } \\
325,000 / \mathrm{mm}^{3} \\
12-24 \mathrm{hrs}=30,000 / \mathrm{mm}^{3}, \\
\text { Day } 2 \text { onwards }=21,000 / \mathrm{mm}^{3}\end{array}$ & \\
\hline Increased or Decreased total ANC & $\begin{array}{l}\text { at birth 1800 0r 5400, } \\
12-48 \text { hrs } 14000, \text { and } \\
48 \text { hrs onwards } 5400\end{array}$ & 1 \\
\hline Immature PMN count (Band form) & $310 \%$ & \\
\hline $\begin{array}{l}\text { Degenerative changes in PMN } \\
\text { (Toxic granulation) }\end{array}$ & Present (3 3) & 1 \\
\hline CRP & Raised $>5 \mathrm{mg} / \mathrm{dl}$ & 1 \\
\hline Platelet count & $150,000 / \mathrm{mm} 3$ & 1 \\
\hline
\end{tabular}

$\mathrm{CRP}=\mathrm{C}$-reactive protein,

ANC $=$ Absolute neutrophil count (neutrophils $\% x$ total WBC count).

$\mathrm{PMN}=$ Polymorphonuclear cell

The investigation for sepsis include complete blood counts, ANC, band form, toxic granulation, vacuolation, platelet count, CRP measurements and blood culture. Findings of HSS were recorded in a proforma and later compared with results of blood cultures.

\section{Interpretation of Hematological Scoring System}

\begin{tabular}{|cc|}
\hline Score & Interpretation \\
\hline$\leq 2$ & Sepsis (very unlikely) \\
\hline$\geq 3$ & Sepsis (considered positive) \\
\hline$>4$ & Sepsis (most likely) \\
\hline
\end{tabular}

The study was conducted on 128 neonates presenting with clinical features suspected of neonatal sepsis. Out of 128 neonates, there were $65.6 \%$ male neonates and $34.4 \%$ female. In this study Nearly half of the newborns (35.1\%) were preterm. On presentation HSS was applied on all neonates with suspected neonatal sepsis and the results were obtained that 32\% had positive HSS and $68 \%$ had negative results. Most of them had raised CRP ( $32 \%$ ) and low platelet count ( $25 \%$ ) (Table II). 
Table II : Hematological Screening Score (HSS)

\begin{tabular}{|l|c|c|}
\hline \multicolumn{1}{|c|}{ Variable } & Frequency & Percentage \\
\hline $\begin{array}{l}\text { White blood cell count } \\
\text { • High }\end{array}$ & 8 & 6.25 \\
- Low & 5 & 3.90 \\
\hline $\begin{array}{l}\text { Absolute neutrophil count } \\
\text { - High }\end{array}$ & 35 & 27.34 \\
low & 3 & 2.34 \\
\hline Immature PMN count (band form) & 13 & 10.15 \\
\hline Degenerative changes in PMN (toxic granule) & 2 & 1.56 \\
\hline Raised C Reactive protein & 41 & 32.03 \\
\hline Low Platelet count & 32 & 25.00 \\
\hline
\end{tabular}

Table III : HSS and Blood Cultures

\begin{tabular}{|c|l|c|c|c|}
\hline \multicolumn{5}{|c|}{ Neonatal Sepsis on HSS * Neonatal Sepsis on Blood Culture } \\
Cross tabulation \\
\hline \multirow{2}{*}{} & \multicolumn{2}{c|}{ Neonatal Sepsis on Blood Culture } & \\
\cline { 2 - 4 } & Positive & Negative & Total \\
\hline \multirow{2}{*}{ Neonatal Sepsis on HSS } & Positive & 10 & 31 & 41 \\
\cline { 2 - 4 } & Negative & 4 & 83 & 87 \\
\hline \multicolumn{2}{|c|}{ Total } & 14 & 114 & 128 \\
\hline
\end{tabular}

Sensitivity $=71 \%$, Specificity $=73 \%$, Positive Predictive Value $=24 \%$, Negative Predictive Value $=95 \%$

After HSS assessment all neonates were underwent blood cultures and neonatal sepsis was seen in $11 \%$ (culture positive) of cases and negative in $89 \%$. On applying the formulae for calculation, sensitivity of HSS was found to be $71 \%$ and specificity $73 \%$. The positive predictive value of HSS was $24 \%$ and negative predictive value was $95 \%$ (Table III).

\section{Discussion}

Despite the advances in neonatal care, early onset neonatal sepsis remains a serious and potentially life-threatening disease. The signs and symptoms of neonatal sepsis may be subtle and nonspecific being clinically indistinguishable from various noninfectious conditions and metabolic disorders. It is the current practice to start empirical antibiotic therapy in all neonates showing infection-like symptoms. However, this may expose newborns to risk of adverse drug effects on one hand and lead to nosocomial complications and the emergence of resistant strains on the other. Accurate and quick diagnosis is therefore essential so that timely treatment of a potentially fatal disease can be provided and at the same time damages deriving from the unnecessary use of antibiotics be prevented. The definitive diagnosis of septicemia is made by a positive blood culture, which requires a minimum of 48-72 hours, yields a positive result in $10-60 \%$ of cases. Blood culture remains the gold standard for diagnosis of neonatal sepsis, yet it is important to develop effective screening tools which can presumably diagnose or exclude neonatal sepsis at the time of presentation and that is why this study was carried out. ${ }^{12}$ Early diagnosis of neonatal septicemia is still a great challenge. For early diagnosis of neonatal septicemia a hematologic scoring system (HSS) of Rodwell was introduced in the past. It included hematological parameters and showed that such score could accurately predict the presence or absence of infection and be reliable. ${ }^{13}$ The HSS assigns a score of 1 for each of seven hematologic findings and shown to be significantly $(P<0.005)$ associated with sepsis. ${ }^{13}$ As no single individual haematological parameter is superior in comparison to another in predicting neonatal sepsis, a combination of these parameters in the form of HSS has been recommended. Hematologic scoring system (HSS) should improve the efficiency of the $\mathrm{CBC}$ as a screening test for sepsis until a reliable diagnostic test is available. The HSS has practical advantages; it is applicable to all infants, including those who have received antibiotic therapy prior to evaluation and simplifies the interpretation of hematologic profile. In this study with a cut off score 3 we observed a sensitivity of $71 \%$, specificity of $73 \%$, PPV 24\%, NPV 95\%. These results were consistent with other studies.14,15 Many studies performed to evaluate the haematological scoring system for the early diagnosis of neonatal sepsis revealed variable results, but all points towards its utility. ${ }^{16}$ Ghosh et al and Narasimha et al reported that Immature PMN count and I:T PMN ratio is sensitive indicator of neonatal sepsis. Degenerative changes in the PMNs made no significant contribution in the diagnosis, in this study. ${ }^{17}$ Presence of toxic granules indicates the production of unusual PMNs during infection and stress induced leucopoiesis. They are never seen in healthy babies. Their presence invariably indicates sepsis, but their count is not always increased. Thrombocytopenia was frequently associated with sepsis and indicated poor prognosis. This is thought to be due to increased platelet destruction, sequestration secondary to infections, failure in platelet production due to reduced megakaryocytes or damaging effects of endotoxin. ${ }^{18,19}$

Higher the score on HSS, more are the chances of sepsis. The simplification and standardization of the interpretation of this global test is still required. Variety of other rapid detection methods of microorganisms, like DNA probes, automated blood culture system and fluorometric detection systems are also available globally, but HSS can still be used as a screening test for diagnosing sepsis and to differentiate infected neonates from the non-infected ones. Furthermore, the sensitivity and the specificity of the test are also high, with certainty of sepsis increasing with the score. ${ }^{15}$ Murphy et al reported on $100 \%$ 
sensitivity and $100 \%$ negative predictive value of two normal white blood cell counts (WBC) within 8 to 12 hours and a negative blood culture at 24 hours for ruling out early-onset sepsis in the neonate. ${ }^{20}$ Measurement of immature neutrophil granulocytes has been considered to be a helpful early indicator of various infectious conditions and has a long clinical tradition in the diagnosis of bacterial sepsis in neonates. ${ }^{21}$ CRP is one of the most widely available; most studied, and most used laboratory tests for neonatal bacterial infection. It is well known that it provides limited sensitivity when determined during the early phases of the disease, especially at the initial presentation, but provides very high negative predictive values and is thus useful for identifying infants unlikely to be infected or monitoring the response to treatment. ${ }^{22}$ Use of CRP in neonatal sepsis is complicated by a nonspecific rise that starts shortly after birth. 23,24

In this study $10.9 \%$ neonates were considered as proven sepsis by blood culture. However suspected sepsis groups comprises a difficult diagnostic group and could not be ignored, because fatal infection had been reported in the presence of negative blood culture. $^{25}$

\section{Conclusion}

Haematological scoring system is a simple, quick, readily available effective tool with high sensitivity and specificity in the early diagnosis of neonatal sepsis.

\section{References}

1. Wynn J, Cornell TT, Wong HR, Shanley TP. The Host Response to Sepsis and Developmental Impact. Pediatrics 2010;125:1031-41.

2. Darmstadt GL, Saha SK, Choi Y, Arifeen SE. Population-based incidence and etiology of community-acquired neonatal bacteremia in Mirzapur, Bangladesh: An Observational Study. J Infect Disease 2009;200:906-15.

3. Rasul $\mathrm{CH}$, Hassan MA, Habibullah M. Neonatal sepsis and use of antibiotic in a tertiary care hospital. Pakistan J Med Science 2007; 23 (1) : 78-81.

4. Vergnano $S$, Sharland $M$, Kazembe $P$, Mwansambo $C$. Neonatal sepsis : an international perspective. Arch Dis Child Fetal Neonatal Edition 2005; 90 : 220-22.

5. Dawodu A, Urman ALK, Danso TK. A case control study of neonatal sepsis: Experience from Saudi Arabia, Journal tropical pediatrics 1997; 43: 84-88.

6. Chowdhury AMAK, Rahman M, Karim AQMR. Characteristics of septicemia in newborn in Dhaka Shishu Hospital. Dhaka Shishu Hospital Journal 1998; 14: 9-12

7. Stoll BJ. Infections of the Neonatal Infant. In: Kliegman RM, Behrman RE, Jenson $\mathrm{HB}$, Stanton BF, editors. Nelson textbook of Pediatrics. 18th ed. Saunders. 2007;794-809.

8. Shirazi H, Riaz S, Tahir R. Role of the Hematological Profile in Early Diagnosis of Neonatal Sepsis. Ann Pak Inst Med Science 2010; 6(3):152-56.

9. Edgar JDM, Gabriel V, Gallimore JR, McMillan SA. A prospective study of the sensitivity, specificity and diagnostic performance of soluble intercellular adhesion molecule, highly sensitive C-reactive protein, soluble E-selectin and serum amyloid $\mathrm{A}$ in the diagnosis of neonatal infection. BMC Pediatrics 2010;10:22-25.

10. Haque KN. Neonatal Sepsis in the Very Low Birth Weight Preterm Infants:Part 2: Review of Definition, Diagnosis and Management. J Med Science 2010; 3(1):11-27.

11. Mishra UK, Jacobs SE, Doyle LW, Garland SM. Newer approaches to the diagnosis of early onset neonatal sepsis. Arch Dis Child Fetal Neonatal Edition 2006; 91:208-12.

12. Fowlie PW, Schmidt B. Diagnostic tests for bacterial infection from birth to 90 days-A systematic review. Arch Dis Child Fetal Neonatal. 1998;78:92-98

13. Rodwell RL, Leslie AL , Tudehope DI. Early diagnosis of neonatal sepsis using a hematologic scoring system. J Pediatrics 1988;112:761-67.

14. Manucha $V$, Rusia U, Sikka M, Faridi MMA. Utility of hematological parameters \& c-reactive protein in the detection of neonatal sepsis. J Paediatr Child Health 2002;38:459-64.

15. Khair KB, Rahman MA, Sultana T, Roy CK, Rahman MQ, Shahidullah M. Role of hematologic scoring system in early diagnosis of neonatal septicemia. BSMMU Journal 2010;3:62-67.

16. Polin RA, Parravicini E, Regan PA. Bacterial sepsis and meningitis. In: Robert MK, Hal BJ, editors. Avery 's disease of the new born. 8th ed. WB Saunders: Philadelphia 2005;551-70.

17. Narasimha A, Kumar ML. Significance of Hematological Scoring System (HSS) in early diagnosis of neonatal sepsis. Indian J Hematol Blood Transfusion 2011;27:14-17.

18. Eissa DS, El-Farrash RA. New insights into thrombopoisis in neonatal sepsis. Platelets 2013;24(2):122-28.

19. Arif SH, Ahmad I, Ali SM, Khan HM. Thrombocytopenia and bacterial sepsis in neonates. Indian J Hematol. Blood Transfusion 2012;28(3):147-51

20. Murphy $\mathrm{K}$, Weiner J. Use of leukocyte counts in evaluation of early-onset neonatal sepsis. Pediatr Infect Dis Journal 2012;31:1-4

21. Buttarello M, Plebani M. Automated blood cell counts: state of the art. Am J Clinical Pathology 2008;130:104-16.

22. Hofer N, Müller W, Resch B. Non-infectious conditions and gestational age influence $\mathrm{C}$-reactive protein values in newborns during the first 3 days of life. Clin Chem Lab Medicine 2011;49:297-302.

23. Chiesa C, Natale F, Pascone R, Osborn JF, Pacifico L. C- reactive protein and procalcitonin: reference intervals for preterm and term newborns during the early neonatal period. Clin Chim Acta 2011; 412: 1053-59.

24. Hofer N, Zacharias E, Muller W, Resch B. An update on use of C-Reactive Protein in early onset neonatal sepsis: Current insights and new tasks. Neonatology 2012; 102:25-36

25. Draz NI, Taha SE, Abou-Shady NM. Comparison of broad range DNA PCR to conventional blood culture for diagnosis of sepsis in the newborn. Egyptian J Med Human Genetics 2013; 14(4):403-11. 